\title{
Co-digestion of Rhodosporidium toruloides biorefinery wastes for biogas production
}

\author{
Isabel Paula Marques ${ }^{\mathrm{a}}$, Ana Paula Batista ${ }^{\mathrm{b}}$, Aldo Coelho ${ }^{\mathrm{a}}$, Teresa Lopes da Silva ${ }^{\mathrm{a}, *}$ \\ a Laboratório Nacional de Energia e Geologia, Unidade de Bioenergia, Estrada do Paço do Lumiar, 1649-038 Lisboa, Portugal \\ b LEAF-Linking Landscape, Environment, Agriculture and Food, Instituto Superior de Agronomia, Universidade de Lisboa, Tapada da Ajuda, 1349-017 Lisboa, Portugal
}

\section{A R T I C L E I N F O}

\section{Keywords:}

R. toruloides

Yeast biorefinery

Wastes mixtures

Co-digestion

Biogas

\begin{abstract}
A B S T R A C T
Rhodosporiodum toruloides NCYC 921 yeast biorefinery is able to simultaneously provide a gaseous energy carrier, lipids and high-value carotenoids, either using glucose or carob pulp syrup as carbon source in the cultivation step for biomass production. The resulting leftover materials from the yeast biorefinery (YR- de-oiled yeast biomass residue, GS- glucose supernatant, CS- carob supernatant and CR- carob residue) were valued through anaerobic digestion in different mixtures, according to the generated effluents volume. Biogas productions of 55, 31 and $29 \mathrm{~mL}$ and methane yield of 236, 179 and $144 \mathrm{~mL} \mathrm{CH}_{4} / \mathrm{g}$ substrate volatile solids (VS) were recorded for the studied mixtures MIX I (YR + GS), MIX II (YR + CS) and MIX III (YR + CS + CR) respectively. The comparison between the biogas production and volatile solids removal obtained for the individual substrates and for the admixtures substrates digestions revealed that co-digestion favoured the conversion of the biorefinery wastes into biogas/methane. Flow cytometry analysis of cells stained with propidium iodide revealed that the proportion of cells with injured membrane was lower in the substrate mixtures digestions than in the individual waste digestions, indicating that co-digestion mitigates the negative effect of potential toxic compounds present in the wastes or produced during the process digestion on the microbial consortium.
\end{abstract}

\section{Introduction}

As the global population continues rising and as the efforts to protect the environment continue to fall short of needs, the drawbacks derived from fossil fuel use are becoming ever and ever more stark and serious. Pollution is a major disadvantage of fossil fuel use due to its contribution to the greenhouse effect and the global warming experienced throughout earth nowadays. Therefore, it is urgent to promote the development of renewable and clean energy sources and strengthen their sustainability.

Microorganisms have been considered a potential renewable source of biofuels and, in particular, oleaginous yeasts have been investigated as a source of biodiesel [1-3]. So far, microbial biofuels are not economically sustainable, thus new approaches are being studied, such as the use of low-cost raw materials as feedstock and/or the coproduction of high added-value products, maximizing the value derived from the whole process, within a biorefinery concept, with a desirable minimal environmental impact. The waste streams derived from these biorefinery processes should therefore be further treated and/or energetically valued in order to accomplish the principles of a circular economy which envisages zero-waste output. Moreover, organic waste should be treated according to the Organic Waste Hierarchy, ensuring proper source-separation and giving priority to composting and biogas generation, after human and animal feed [4].

Anaerobic digestion is a biological process which enables the recovery of renewable energy/biogas from wastes in a controlled and efficient way, providing power generation or fuel gas [5] and, additionally, supplies a digested flow which can be used for irrigation and soil fertilization. However, there may be some drawbacks from anaerobic digestion processes, related to the recalcitrant and toxic properties and unbalanced composition of many organic effluents. Current methods involving anaerobic treatment are only applicable if using high dilutions and pre-treatments, further compromising the process economy with the use of chemicals required to obtain, for instance, a proper alkalinity or nitrogen content. A "complementary substrate" concept has been developed as a feeding strategy, in order to improve the recalcitrant and toxic effluent conversion, such as olive mill wastewater, avoiding substrate pre-treatments $[6,7]$. The admixture with another complementary effluent is a way to obtain load and toxicity dilutions but also a way to compensate the system for unbalanced

\footnotetext{
* Corresponding author.

E-mail address: teresa.lopessilva@lneg.pt (T. Lopes da Silva).
} 